\title{
General Adaptive Neighborhood Image Restoration, Enhancement and Segmentation
}

\author{
Johan Debayle, Yann Gavet, and Jean-Charles Pinoli \\ Ecole Nationale Supérieure des Mines de Saint-Etienne \\ Centre Ingénierie et Santé (CIS), Laboratoire LPMG, UMR CNRS 5148 \\ 42023 Saint-Etienne Cedex 2, France
}

\begin{abstract}
This paper aims to outline the General Adaptive Neighborhood Image Processing (GANIP) approach [1-3], which has been recently introduced. An intensity image is represented with a set of local neighborhoods defined for each point of the image to be studied. These so-called General Adaptive Neighborhoods (GANs) are simultaneously adaptive with the spatial structures, the analyzing scales and the physical settings of the image to be addressed and/or the human visual system. After a brief theoretical introductory survey, the GANIP approach will be successfully applied on real application examples in image restoration, enhancement and segmentation.
\end{abstract}

\section{The General Adaptive Neighborhood (GAN) Paradigm}

This paper deals with 2D intensity images, that is to say image mappings defined on a spatial support $D$ in the Euclidean space $\mathbb{R}^{2}$ and valued into a gray tone range, which is a real numbers interval. The General Adaptive Neighborhood paradigm has been introduced in order to propose an original image representation for adaptive processing and analysis. The central idea is the notion of adaptivity which is simultaneously associated to the analyzing scales, the spatial structures and the intensity values of the image to be addressed.

\subsection{Adaptivity with Analyzing Scales}

A multiscale image representation such as wavelet decomposition [4] or isotropic scale-space [5], generally takes into account analyzing scales which are global and a priori defined, that is to say extrinsic scales. This kind of multiscale analysis presents a main drawback since a priori knowledge, relating to the features of the studied image, is consequently required. On the contrary, an intrinsic multiscale representation such as anisotropic scale-space [6], takes advantage of scales which are self-determined by the local image structures. Such a decomposition does not need any a priori information. 


\subsection{Adaptivity with Spatial Structures}

The image processing techniques using spatially invariant transformations, with fixed operational windows, give efficient and compact computing structures, in the sense where data and operators are independant. Nevertheless, they consequently have several drawbacks such as creating artificial patterns, changing the detailed parts of large objects, damaging transitions or removing significant details. Alternative approaches towards context depend processing have been proposed [7]. A spatially adaptive image processing implies that operators are no longer spatially invariant, but must vary over the whole image with adaptive windows, taking locally into account the image context.

\subsection{Adaptivity with Intensity Values}

In order to develop powerful image processing operators, it is necessary to represent intensity images within mathematical frameworks (most of the time of a vectorial nature) based on a physically and/or psychophysically relevant image formation process. In addition, their mathematical structures and operations (the vector addition and then the scalar multiplication) have to be consistent with the physical nature of the images and/or the human visual system, and computationally effective. Thus, although the Classical Linear Image Processing Framework (CLIP), i.e. with the usual vectorial operations, has played a central role in image processing, it is not necessarily the best choice. Indeed, it was shown [8] that the usual addition is not a satisfactory solution in some non-linear physical settings, such as that based on multiplicative or convolutive image formation model. The reasons are that the classical addition operation and consequently the usual scalar multiplication are not consistent with the combination and amplification laws to which such physical settings obey. However, using the power of abstract linear algebra, it is possible to go up to the abstract level and to explore General Linear Image Processing (GLIP) frameworks [9, 10], in order to include situations in which signals or images are combined by processes other than the usual vector addition. Consequently, operators based on such intensity-based image processing frameworks should be consistent with the physical and/or physiological settings of the images to be processed. For instance, the Logarithmic Image Processing (LIP) framework of intensity images $(f, g, \ldots)$ has been introduced $[11,12]$ with its vector addition $\mathbb{A}$, its vector subtraction $\Delta$ and its scalar multiplication $\Delta$ defined respectively as following:

$$
\begin{aligned}
& f \triangle g=f+g-\frac{f g}{M} \\
& f \triangle g=M\left(\frac{f-g}{M-g}\right) \\
& \alpha \triangle f=M-M\left(1-\frac{f}{M}\right)^{\alpha}, \quad \alpha \in \mathbb{R}
\end{aligned}
$$

where $M \in \mathbb{R}$ denotes the upper bound of the range where intensity images are digitized and stored. 
The LIP framework has been proved to be consistent with the transmittance image formation model, the multiplicative reflectance image formation model, the multiplicative transmittance image formation model, and with several laws and characteristics of human brightness perception [10,13].

\section{GANs Sets}

In the so-called General Adaptive Neighborhood Image Processing (GANIP) approach [1-3], a set of General Adaptive Neighborhoods (GANs set) is identified about each point in the image to be analyzed. A GAN is a subset of the spatial support constituted by connected points whose measurement values, in relation to a selected criterion (such as luminance, contrast, thickness, ... .), fit within a specified homogeneity tolerance. These GANs are used as adaptive windows for image transformations or quantitative image analysis.

The space of image (resp. criterion) mappings, defined on the spatial support $D$ and valued in a real numbers interval $\tilde{E}$ (resp. $E$ ), is represented in a GLIP framework, denoted $\mathcal{I}$ (resp. $\mathcal{C}$ ). The GLIP framework $\mathcal{I}$ (resp. $\mathcal{C}$ ) is then supplied with an ordered vectorial structure, using the formal vector addition $\tilde{\oplus}$ (resp. $\oplus$ ), the formal scalar multiplication $\tilde{\otimes}($ resp. $\otimes)$ and the classical total order relation $\geq$ defined directly from those of real numbers:

$$
\forall(f, g) \in \mathcal{I}^{2} \text { or } \mathcal{C}^{2} \quad f \geq g \Leftrightarrow(\forall x \in D \quad f(x) \geq g(x))
$$

There exists several GANs sets, whose each collection satisfies specific properties [1]. This paper presents the most elementary kind of these ones, denoted $V_{m_{\square}}^{h}(x)$. For each point $x \in D$ and for an image $f \in \mathcal{I}$, the GANs $V_{m_{\square}}^{h}(x)$ are included as subsets in $D$. They are built upon a criterion mapping $h \in \mathcal{C}$ (based on a local measurement such as luminance, contrast, thickness, ... related to $f$ ), in relation with an homogeneity tolerance $m_{\square}$ belonging to the positive intensity value range $E^{\oplus}$. More precisely, $V_{m_{\square}}^{h}(x)$ is a subset of $D$ which fulfills two conditions :

1. its points have a measurement value close to that of the point $x: \forall y \in$ $V_{m_{\square}}^{h}(x) \quad|h(y) \ominus h(x)|_{\emptyset} \leq m_{\square}$, where $\theta$ and $|\cdot|_{\bigcirc}$ denote the considered GLIP subtraction and GLIP modulus, respectively,

2. the set is path-connected (with the usual Euclidean topology on $D \subseteq \mathbb{R}^{2}$ ).

The GANs are thus defined as following:

$$
\forall\left(m_{\square}, h, x\right) \in E^{+} \times \mathcal{C} \times D \quad V_{m_{\square}}^{h}(x)=C_{h^{-1}\left(\left[h(x) \ominus m_{\square}, h(x) \oplus m_{\square}\right]\right)}(x)
$$

where $C_{X}(x)$ denotes the path-connected component (with the usual Euclidean topology on $D \subseteq \mathbb{R}^{2}$ ) of $X \subseteq D$ containing $x \in D$.

Figure 1 gives a visual impression, on a 1-D example, of the computation of a GAN in the LIP framework (i.e. with the $\triangle$ vector addition (1) and the $\triangle$ vector subtraction (2)). 


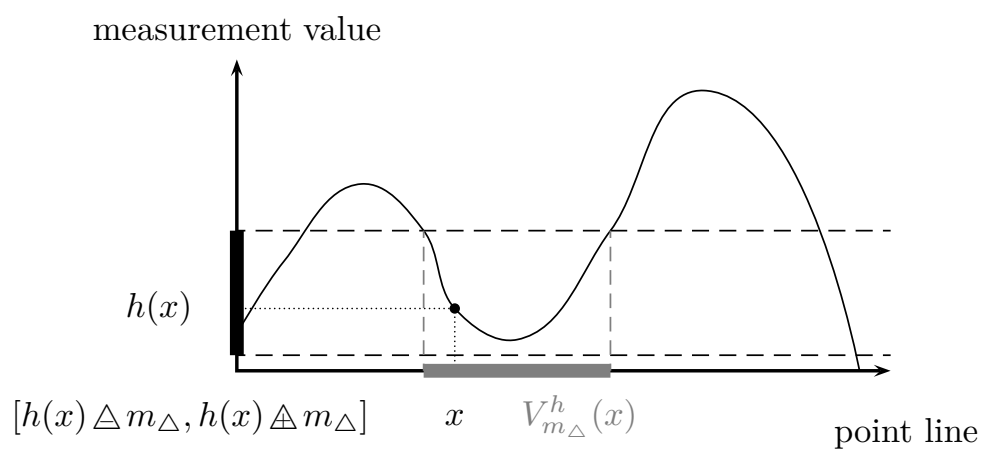

Fig. 1. One-dimensional computation of an adaptive neighborhood set $V_{m_{\triangle}}^{h}(x)$ in the LIP framework. For a point $x$, a tube of tolerance $m_{\square}$ is first computed around $h(x)$. Secondly, the inverse map of this interval gives a subset of the 1-D spatial support. Finally, the path-connected component holding $x$ provides the GAN $V_{m_{\triangle}}^{h}(x)$.

\section{GAN Mean and Rank Filtering}

Usual image to image transformations generally work on fixed-size and fixedshape operational windows, either they are convolution filters (mean, ... ) or rank operators (min, max, median, ... ). This kind of operators removes thin details and displaces contours. In the GANIP approach, adaptive filters are introduced in substituting the usual disks $B_{r}($.$) of radius r$ as isotropic operational windows by the anisotropic GANs $V_{m_{\square}}^{h}($.$) (Fig. 2).$

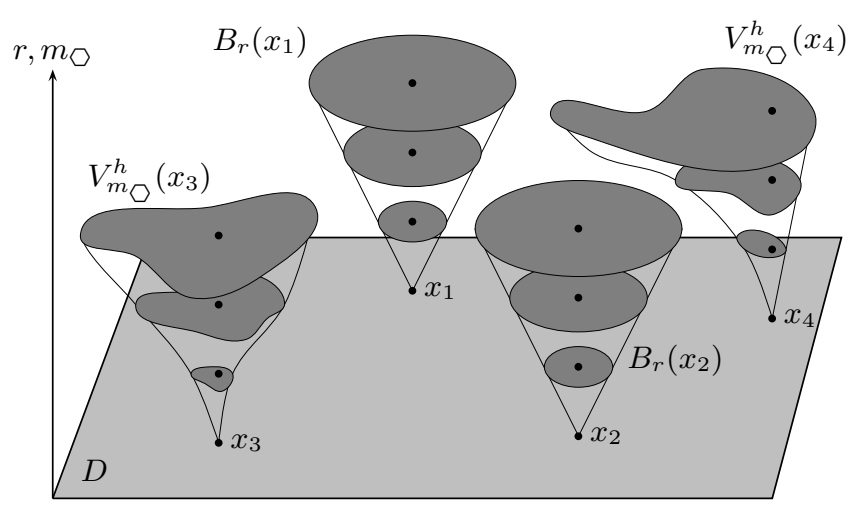

Fig. 2. Example of adaptive $V_{m_{\bigcirc}}^{h}($.$) and non-adaptive B_{r}($.$) operational windows with$ three values both for the homogeneity tolerance parameter $m_{\square}$, and for the disks radius $r$. The shape of $B_{r}\left(x_{1}\right)$ and $B_{r}\left(x_{2}\right)$ are identical and $\left\{B_{r}(x)\right\}_{r}$ is a family of homothetic sets for each point $x \in D$. On the contrary, the shape of $V_{m_{\square}}^{h}\left(x_{3}\right)$ and $V_{m_{\square}}^{h}\left(x_{4}\right)$ are dissimilar and $\left\{V_{m_{\square}}^{h}(x)\right\}_{m}$ is not a family of homothetic sets. 
The resulting GANIP-based operators perform consistent image processing, such as adaptive mean, median or morphological filters which are introduced in the following. Mean and rank filtering are simple, intuitive and easy to implement methods for smoothing images, i.e. reducing the amount of intensity variation between one pixel and the next. They are often used to reduce noise effects in images [14].

The idea of mean filtering consists in replacing the gray tone of every point in an image with the mean ('average') gray tone of its neighbors, including itself. This has the effect of eliminating point values which are unrepresentative of their surroundings. Mean filtering is usually thought of as a convolution filter. Like other convolutions it is based around a kernel, which represents the shape and size of the neighborhood to be sampled when calculating the mean. Often an isotropic kernel is used, as a disk of radius 1, although larger kernels (e.g. disk of radius 2) can be used for more severe smoothing. (Note that a small kernel can be applied more than once in order to produce a similar - but not identical - effect as a single pass with a large kernel).

Rank filters in image processing sort (rank) the gray tones in some neighborhood of every point in ascending order, and replace the seed point by some value $k$ in the sorted list of gray tones. When performing the well-known median filtering [14], each point to be processed is determined by the median value of all points in the selected neighborhood. The median value $k$ of a population (set of points in a neighborhood) is that value for which half of the population has smaller values than $k$, and the other half has larger values than $k$.

So, the GANs mean and rank filters are introduced by substituting the isotropic neighborhoods, generally used for this kind of filtering, with the (anisotropic) general adaptive neighborhoods (GANs).

\section{GAN Mathematical Morphology}

Mathematical Morphology (MM) [15] is an important and nowadays a traditional theory in image processing. Its development leads to several image processing tools that are extremely useful in image enhancement, image segmentation and classification, pattern recognition, texture analysis and synthesis. The elementary morphological operators of dilation and erosion use an operational window named Structuring Element (SE). Generally, the SEs are extrinsically defined and have consequently fixed shape and size.

\subsection{Adaptive Structuring Elements}

The basic idea in the General Adaptive Neighborhood Mathematical Morphology (GANMM) is to replace the usual SEs by GANs, providing adaptive operators and filters. More precisely the Adaptive Structuring Elements (ASEs), denoted $R_{m_{\square}}^{h}(x)$, are defined as following:

$$
\forall\left(m_{\square}, h, x\right) \in E^{\oplus} \times \mathcal{C} \times D \quad R_{m_{\square}}^{h}(x)=\bigcup_{z \in D}\left\{V_{m_{\square}}^{h}(z) \mid x \in V_{m_{\square}}^{h}(z)\right\}
$$


The GANs $V_{m_{\square}}^{h}(x)$ are not directly used as ASEs, because they do not satisfy the symmetry property contrary to the $R_{m_{\square}}^{h}(x): x \in R_{m_{\square}}^{h}(y) \Leftrightarrow y \in R_{m_{\square}}^{h}(x)$. This symmetry condition is relevant for visual, topological, morphological and practical reasons as explained in [3].

\subsection{Adaptive Morphological Operators}

The elementary dual operators of adaptive dilation and adaptive erosion are defined accordingly to the ASEs:

$$
\begin{aligned}
\forall\left(m_{\square}, h, f\right) \in E^{\oplus} \times \mathcal{C} \times \mathcal{I} \\
\mathrm{D}_{m_{\square}}^{h}(f):\left\{\begin{array}{l}
D \rightarrow \tilde{E} \\
x \mapsto \sup _{w \in R_{m}^{h} \square} f(x)
\end{array}\right. \\
\mathrm{E}_{m_{\square}}^{h}(f):\left\{\begin{array}{l}
D \rightarrow \tilde{E} \\
x \mapsto \inf _{w \in R_{m}^{h}(x)} f(w)
\end{array}\right.
\end{aligned}
$$

Then, several adaptive morphological filters can be defined by combination of these two elementary adaptive morphological operators, in particular:

- adaptive closing: $\mathrm{C}_{m_{\bigcirc}}^{h}(f)=\mathrm{E}_{m_{\bigcirc}}^{h} \circ \mathrm{D}_{m_{\bigcirc}}^{h}(f)$

- adaptive opening: $\mathrm{O}_{m_{\square}}^{h}(f)=\mathrm{D}_{m_{\square}}^{h} \circ \mathrm{E}_{m_{\square}}^{h}(f)$

- adaptive closing-opening: $\mathrm{CO}_{m_{\square}}^{h}(f)=\mathrm{C}_{m_{\square}}^{h} \circ \mathrm{O}_{m_{\square}}^{h}(f)$

- adaptive opening-closing: $\mathrm{CO}_{m_{\square}}^{h}(f)=\mathrm{C}_{m_{\square}}^{h} \circ \mathrm{O}_{m_{\square}}^{h}(f)$

Those resulting GAN morphological operators perform a really spatiallyadaptive image processing and notably, in several and important practical cases, are connected $[3,1]$, which is a great advantage compared to the usual ones that fail to this property.

\section{Practical Application Examples}

Most of the time, image filtering is a necessary step in image pre-processing, such as restoration, pre-segmentation, enhancement, sharpening, brightness correction, ... The GAN-based filtering allows such transformations to be defined $[16,2]$. Three results are here exposed in image restoration, image enhancement and image segmentation.

\subsection{Image Restoration}

This section addresses the image restoration area with a concrete application example in visual image denoising. The aim is to suppress noise as much as possible while preserving image features. Figure 3 exposes results of a denoising 
process applied on the Edouard Manet's painting 'Le Fifre'. It is realized both with classical mean filters, denoted $\mathrm{Mean}_{r}$, using disks of radius $r$ as classical operational windows, and with GAN mean filters, denoted Mean $m_{m_{\Delta}}^{f}$, using GANs computed with the luminance criterion in the LIP framework.

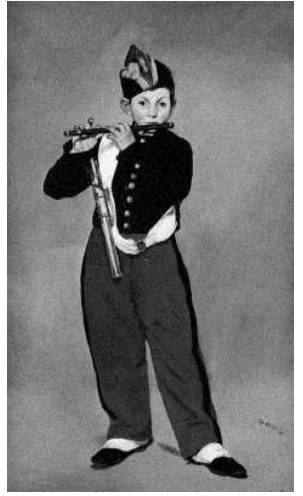

(a) original $f$ image

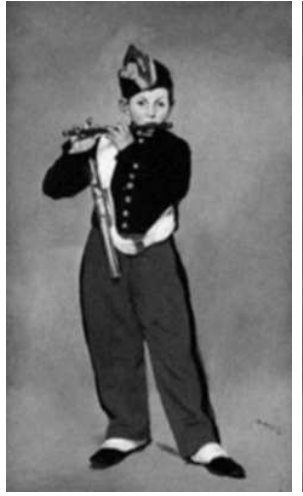

(b) $\operatorname{Mean}_{1}(f)$

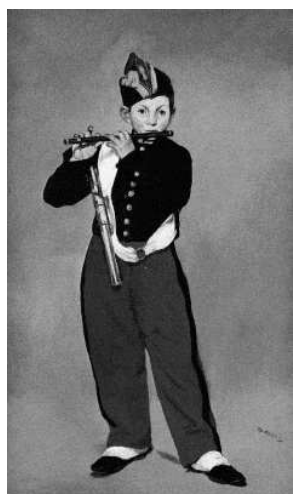

(e) $\operatorname{Mean}_{10 \triangle}^{f}(f)$

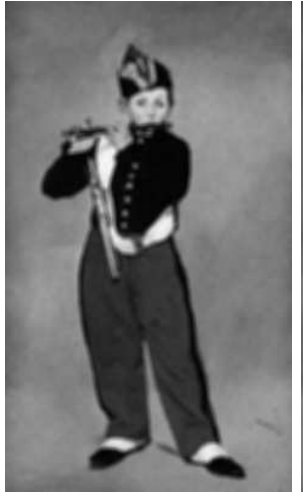

(c) $\operatorname{Mean}_{2}(f)$

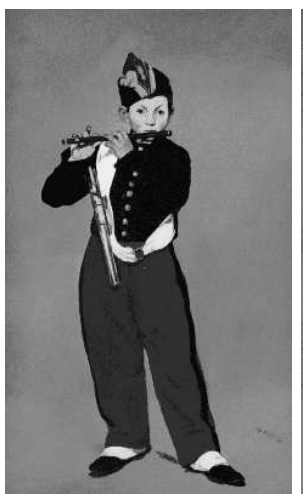

(f) $\operatorname{Mean}_{30_{\triangle}}^{f}(f)$

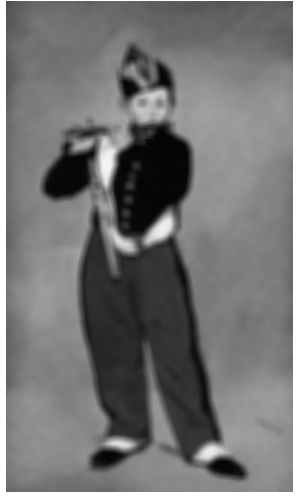

(d) $\operatorname{Mean}_{3}(f)$

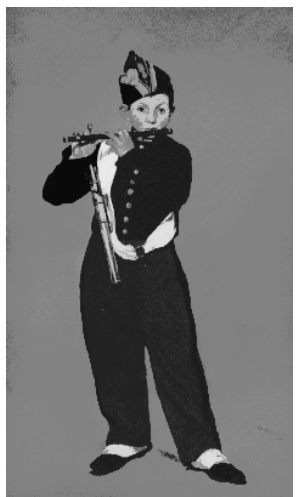

(g) $\operatorname{Mean}_{50 \triangle}^{f}(f)$

Fig. 3. Image restoration through usual (b-d) and adaptive (b-d) mean filtering applied on the original (a) image. The adaptive filters (connected) do not damage edges contrary to the classical filters which blur the image.

Those adaptive filters using the elementary GANs work well if the processed images are noise free or a bit corrupted .

In the presence of impulse noise, such as salt and pepper noise, the GANs need to be combined so as to provide efficient filtering operators [2]. Indeed, the elementary GAN of a corrupted point by such a noise is generally not representative of the region of which it belongs, for any homogeneity tolerance value $m_{\square}$. 


\subsection{Image Enhancement}

Image enhancement is the improvement of image quality [14], wanted e.g. for visual inspection or for machine analysis. Physiological experiments have shown that very small changes in luminance are recognized by the human visual system in regions of continuous gray tones, and not at all seen in regions of some discontinuities [17]. Therefore, a design goal for image enhancement is often to smooth images into more uniform regions, while preserving edges. On the other hand, it has also been shown that somehow degraded images with enhancement of certain features, e.g. edges, can simplify image interpretation both for a human observer and for machine recognition [17]. A second design goal, therefore, is image sharpening [14].

In this paper, the considered image enhancement technique is an edge sharpening process: the approach is similar with unsharp masking [18] type enhancement where a high pass portion is added to the original image. The contrast enhancement process is realized through the toggle contrast [19], whose operator $\kappa_{r}$ is defined in the following:

$$
\begin{aligned}
\forall(f, x, r) \in \mathcal{I} \times D \times \mathbb{R}^{+} \\
\kappa_{r}(f)(x)=\left\{\begin{array}{l}
\mathrm{D}_{r}(f)(x) \text { if } \mathrm{D}_{r}(f)(x)-f(x)<f(x)-\mathrm{E}_{r}(f)(x) \\
\mathrm{E}_{r}(f)(x) \text { otherwise }
\end{array}\right.
\end{aligned}
$$

where $\mathrm{D}_{r}$ and $\mathrm{E}_{r}$ denote the classical dilation and erosion, respectively, using a disk of radius $r$ as structuring element.

This (non-adaptive) toggle contrast will be compared with the adaptive LIP toggle contrast, using a 'contrast' criterion. This transformation requires a 'contrast' definition which is introduced in the digital setting of the LIP framework [20]: The LIP contrast at a point $x \in D$ of an image $f \in \mathcal{I}$, denoted $C(f)(x)$, is defined with the help of the gray values of its neighbors included in a disk $V(x)$ of radius 1 , centered in $x$ :

$$
C(f)(x)=\frac{1}{\# V(x)} \otimes \sum_{y \in V(x)}^{\oplus}(\max (f(x), f(y)) \triangle \min (f(x), f(y)))
$$

where $\sum^{A}$ and \# denote the sum in the LIP sense [20], and the cardinal symbol,
respectively.

Consequently, the so-called adaptive toggle LIP contrast is the transformation $\kappa_{m_{\Delta}}^{C(f)}$, where $C(f)$ and $m_{\triangle}$ represent the criterion mapping and the homogeneity tolerance within the LIP framework (required for the GANs definition), respectively. It is defined as following:

$$
\begin{aligned}
& \forall\left(f, x, m_{\triangle}\right) \in \mathcal{I} \times D \times E^{\Delta} \\
& \kappa_{m_{\Delta}}^{C(f)}(f)(x)=\left\{\begin{array}{l}
\mathrm{D}_{m \Delta}^{C(f)}(f)(x) \text { if } \mathrm{D}_{m_{\Delta}}^{C(f)}(f)(x)-f(x)<f(x)-\mathrm{E}_{m_{\Delta}}^{C(f)}(f)(x) \\
\mathrm{E}_{\Delta}^{C(f)}(f)(x) \text { otherwise }
\end{array}(11)\right.
\end{aligned}
$$


where $\mathrm{D}_{m_{\Delta}}^{C(f)}$ and $\mathrm{E}_{m_{\Delta}}^{C(f)}$ denote the adaptive dilation and adaptive erosion, respectively, using ASEs computed on the criterion mapping $C(f)$ with the homogeneity tolerance $m_{\triangle}$.

Figure 4 illustrates an application example of image enhancement through usual and adaptive toggle contrast, respectively. The process is applied on a real image acquired on the retina of a human eye.
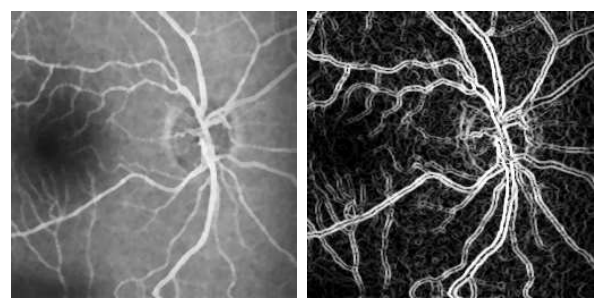

(a) original image $f$ (b) LIP contrast mapping $C(f)$

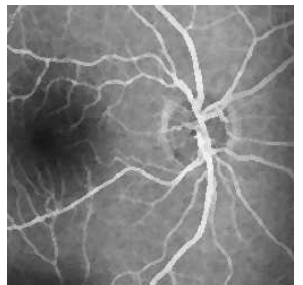

(c) $\kappa_{1}(f)$

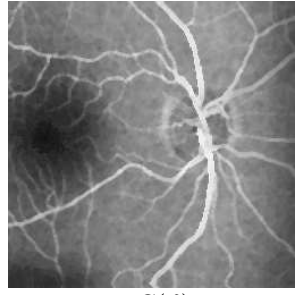

(g) $\kappa_{4 \triangle}^{C(f)}(f)$

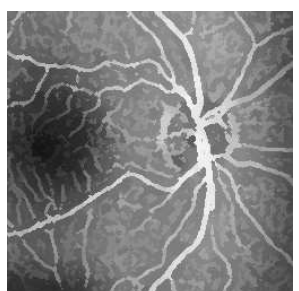

(d) $\kappa_{5}(f)$

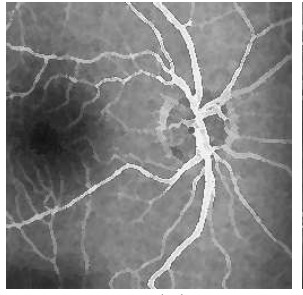

(h) $\kappa_{30 \Delta}^{C(f)}(f)$

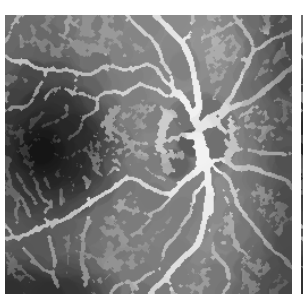

(e) $\kappa_{10}(f)$

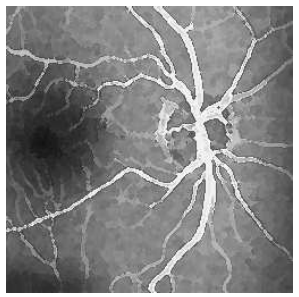

(i) $\kappa_{50 \triangle}^{C(f)}(f)$

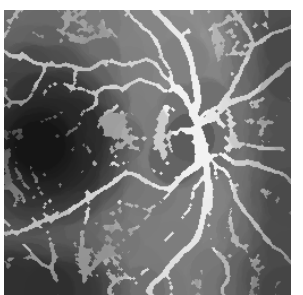

(f) $\kappa_{20}(f)$

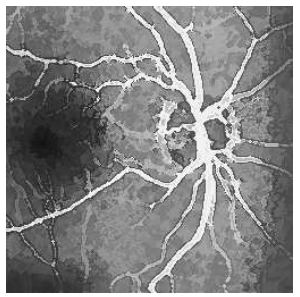

(j) $\kappa_{70 \triangle}^{C(f)}(f)$

Fig. 4. Image enhancement through the toggle contrast process. The operator is applied on a real (a) image acquired on the retina of a human eye. The enhancement is achieved with the usual toggle contrast (c-f) and the GANIP-based toggle LIP contrast (g-j). Using the usual toggle contrast, the edges are disconnected as soon as the filtering becomes too strong. On the contrary, such structures are preserved and sharpened with the GAN filters.

This image enhancement application example confirms that the GANIP operators are more effective than the corresponding classical ones. Indeed, the 
adaptive toggle LIP contrast performs a locally accurate image enhancement, taking into account the notion of contrast within spatial structures of the image. Consequently, only the transitions are sharpened while preserving the homogeneous regions. On the contrary, the usual toggle contrast enhances the image in a uniform way. Thus, the spatial zones around transitions are rapidly damaged as soon as the filtering becomes too strong.

\subsection{Image Segmentation}

The segmentation of an intensity image can be defined as its partition (in fact the partition of the spatial support $D$ ) into different connected regions, relating to an homogeneity condition [14]. In this paper, the segmentation process is based on a morphological transformation called watershed [21] and a GANIP-based decomposition process. It will be illustrated on a human corneal endothelial image provided by the University Hospital Center of Saint-Etienne in France.

The cornea is the transparent surface in the front of the eye. It has a role of protection of the eye, and with the lens, of focusing light into the retina. It is constituted of several layers, such as the epithelium (at the front of the cornea), the stroma and the endothelium (at the back of the cornea). The endothelium contains non-regenerative cells tiled in a monolayer and hexagonal mosaic. This layer pumps water from the cornea, keeping it clear. A high cell density and a regular morphometry of this layer characterize the good quality of a cornea before transplantation, the most common transplantation in the world. Herein lays the importance of the endothelial control. Ex vivo controls are done by optical microscopy on corneal button before grafting. That image acquisition equipment give gray tones images which are segmented, for example by the SAMBA $^{\mathrm{TM}}$ software [22], into regions representing cells. These ones are used to compute statistics in order to quantify the corneal quality before transplantation.

The authors proposed a GANIP-based approach to segment the cornea cells. The process is achieved by a closing-opening morphological filtering using the GAN sets with the luminance criterion in the CLIP framework, followed by a watershed transformation, denoted $W$. A comparison with the results provided by the SAMBA ${ }^{\mathrm{TM}}$ software, whose process is achieved by thresholding, filtering and skeletonization [22], is proposed (Fig. 5). The parameter $m_{\square}$ of the adaptive morphological filter has been tuned to visually provide the best possible segmentation.

The detection process achieved by the GANIP-based morphological approach provides better results (from the point of view of ophthalmologists) than the SAMBA $^{\mathrm{TM}}$ software. Those results highlight the spatially-variant adaptivity of the GANIP-based operators. A more specific study should be investigated for this promising cells detection process. 


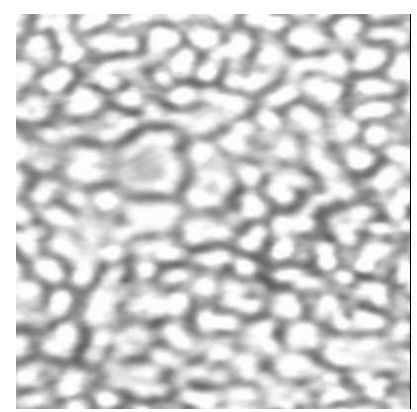

(a) original image $f$

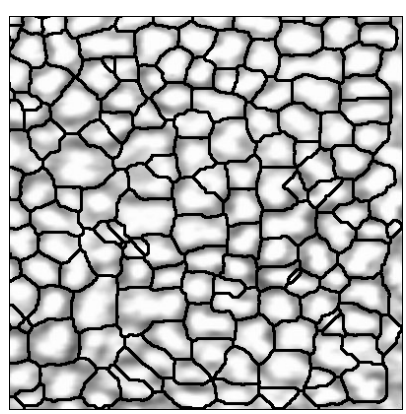

(b) $\mathrm{SAMBA}^{\mathrm{TM}}$ result

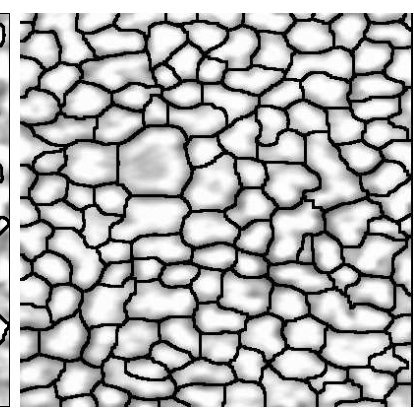

(c) $W\left(\mathrm{CO}_{10}^{f}(f)\right)$

Fig. 5. Segmentation of human endothelial cornea cells (a). The process achieved by the GANIP-based morphological approach (c) provides better results (from the point of view of ophthalmologists) than the SAMBA ${ }^{\mathrm{TM}}$ software [22] (b).

\section{Conclusion and Prospects}

The General Adaptive Neighborhood Image Processing (GANIP) approach allows efficient image processing operators to be built. The GAN-based representation of an image is simultaneously adaptive with its analyzing scales, its spatial structures and its intensity values. In this way, the resulting adaptive operators are spatially variant, relevant from a physical and/or psychophysical point of view and are intrinsically multiscale in the sense where processing scales are locally determined by the image context. These theoretical aspects have been practically confirmed on real application examples in image restoration, enhancement and segmentation. From a practical point of view, the computation of the GANs sets increases the running time of the adaptive operators [3]. Currently, the authors work on GANIP-based topological approaches.

\section{Acknowledgments}

The authors wish to thank their colleague P. Gain from the University Hospital Center of Saint-Etienne in France, who has kindly provided the human corneal endothelial images (Fig. 5-a,b).

\section{References}

1. Debayle, J., Pinoli, J.C.: General Adaptive Neighborhood Image Processing - Part I: Introduction and Theoretical Aspects. (Journal of Mathematical Imaging and Vision) paper accepted on January 26, 2006.

2. Debayle, J., Pinoli, J.C.: General Adaptive Neighborhood Image Processing - Part II: Practical Application Examples. (Journal of Mathematical Imaging and Vision) paper accepted on January 26, 2006. 
3. Debayle, J., Pinoli, J.C.: Spatially Adaptive Morphological Image Filtering using Intrinsic Structuring Elements. Image Analysis and Stereology 24(3) (2005) 145158

4. Mallat, S.G.: A Theory for Multiresolution Decomposition : The Wavelet Representation. IEEE Transactions on Pattern Analysis and Machine Intelligence 11 (1989) 674-693

5. Lindeberg, T.: Scale-Space Theory: a Basic Tool for Analysing Structures at Different Scales. Journal of Applied Statistics 21(2) (1994) 225-270

6. Perona, P., Malik, J.: Scale-Space and Edge Detection using Anisotropic Diffusion. IEEE Transactions on Pattern Analysis and Machine Intelligence 12(7) (1990) 629-639

7. Gordon, R., Rangayyan, R.M.: Feature Enhancement of Mammograms using Fixed and Adaptive Neighborhoods. Applied Optics 23(4) (1984) 560-564

8. Rosenfeld, A.: Picture Processing by Computers. Academic Press, New-York, U.S.A. (1969)

9. Oppenheim, A.V.: Generalized Superposition. Information and Control 11 (1967) $528-536$

10. Pinoli, J.C.: A General Comparative Study of the Multiplicative Homomorphic, Log-Ratio and Logarithmic Image Processing Approaches. Signal Processing 58 (1997) 11-45

11. Jourlin, M., Pinoli, J.C.: A model for logarithmic image processing. Journal of Microscopy 149 (1988) 21-35

12. Jourlin, M., Pinoli, J.C.: Logarithmic Image Processing : The Mathematical and Physical Framework for the Representation and Processing of Transmitted Images. Advances in Imaging and Electron Physics 115 (2001) 129-196

13. Pinoli, J.C.: The Logarithmic Image Processing Model : Connections with Human Brightness Perception and Contrast Estimators. Journal of Mathematical Imaging and Vision 7(4) (1997) 341-358

14. Gonzalez, R.C., Woods, R.E.: Digital Image Processing. Addison-Wesley (1992)

15. Serra, J.: Image Analysis and Mathematical Morphology. Academic Press, London, U.K. (1982)

16. Debayle, J., Pinoli, J.C.: Multiscale Image Filtering and Segmentation by means of Adaptive Neighborhood Mathematical Morphology. In: Proc. of the IEEE ICIP. Volume III., Genova, Italy (2005) 537-540

17. Stockham, T.G.: Image Processing in the Context of a Visual Model. In: Proceedings of the IEEE. Volume 60. (1972) 825-842

18. Ramponi, G., Strobel, N., Mitra, S.K., Yu, T.H.: Nonlinear Unsharp Masking Methods for Image-Contrast Enhancement. Journal of Electronic Imaging 5(3) (1996) 353-366

19. Soille, P.: Filtering. In: Morphological Image Analysis. Principles and Applications. Springer Verlag, New York (2003) 241-266

20. Jourlin, M., Pinoli, J.C., Zeboudj, R.: Contrast definition and contour detection for logarithmic images. Journal of Microscopy 156 (1988) 33-40

21. Beucher, S., Lantuejoul, C.: Use of watersheds in contour detection. In: International Workshop on image processing, real-time edge and motion detection/estimation, Rennes, France (1979)

22. Gain, P., Thuret, G., Kodjikian, L., Gavet, Y., Turc, P.H., Theillere, C., Acquart, S., LePetit, J.C., Maugery, J., Campos, L.: Automated tri-image analysis of stored corneal endotheliun. British Journal of Ophtalmology 86 (2002) 801-808 